\title{
Metabólitos Secundários e ação antioxidante de Croton heliotropiifolius e Croton blanchetianus
}

\author{
Vitória Viviane Ferreira de Aquino ${ }^{a^{*}}$, José Galberto Martins da Costab ${ }^{\mathrm{b}}$ Elissandra Couras Angélicoc, Rosália \\ Severo de Medeiros ${ }^{d}$, Maria de Fátima de Araújod, Onaldo Guedes Rodriguesa \\ a Programa de Pós-Graduação em Ciência Animal, Universidade Federal de Campina Grande, Patos, Paraíba, 58700-971, Brasil. \\ *onaldoguedesrodrigues@yahoo.com.br \\ ${ }^{b}$ Laboratório Multiusuário de Pesquisas Ambientais, Universidade Federal de Campina Grande, Patos, Paraíba, 58701-971, Brasil. \\ ${ }^{c}$ Laboratório de Pesquisa de Produtos Naturais, Crato, Ceará, 63105-000, Brasil. \\ d Unidade Acadêmica de Ciências Biológicas, Universidade Federal de Campina Grande, Patos, 58700-971, Brasil.
}

Recebido: 21 março 2017 / Aceito: 30 maio 2017 / Publicado online: 27 setembro 2017

\begin{abstract}
Resumo
Antioxidantes são compostos que reagem com os radicais livres controlando o estresse oxidativo. Muitas doenças estão associadas à produção de radicais livres, os quais tem estimulado investigações do potencial antioxidante de diversas famílias da flora mundial. Essa pesquisa teve como objetivo identificar as principais classes de metabólitos secundários presentes nos estratos etanólicos das folhas de Croton blanchetianus (Marmeleiro) e Croton heliotropiifolius (Velame), e avaliar o seu potencial antioxidante. Os estratos etanólicos foram obtidos das folhas, apresentou um rendimento de 3,29\% e 3,26\%. Em seguida, os estratos foram submetidos a análise fitoquímica, nos quais foi possível identificar a presença de taninos condensados, flavonoides, flavononas, flavonóis, flavononóis, catequinas e xantonas. A atividade antioxidante dos estratos foi determinada pelo método fotocolorimétrico in vitro realizada por meio do sequestro de radicais livres, usando o DPPH (1,1-difenil-2-picrilhidraliza). Ambos os estratos foram capazes de sequestrar o radical livre DPPH, sendo que o estrato de Croton blanquetianus mostrou ser mais eficiente do que o BHT (Hidroxitolueno butilado; controle positivo), com $\mathrm{CE}_{50}=6,5 \pm 0,5 \mathrm{mg} \mathrm{mL}^{-1}$. Contudo, os resultados observados para os estratos etanólicos, possivelmente sejam atribuídas à presença e a concentração de compostos fenólicos pertencentes as classes dos taninos e flavonoides, os quais foram identificados nesse estudo.
\end{abstract}

Palavras-chave: Radicais livres, fitoquímica, fotocolorimétrico

\section{Secondary metabolites and antioxidant action of Croton heliotropiifolius and Croton blanchetianus}

\begin{abstract}
Antioxidants are compounds that, at optimal concentrations in relation to oxidable substrates, react with free radicals preventing or reducing oxidative stress. Many diseases and degenerative processes are associated with overproduction of free radicals. This stimulated several research groups to investigate the antioxidant potential of substances produced by several families of the world flora. This study identified the major classes of secondary metabolites present in ethanol leaf extracts of $C$. blanchetianus (Marmeleiro) and C. heliotropiifolius (Velame), and assessed their antioxidant potential. Ethanol extracts were obtained by the exhaustive extraction method cold and resulted in 3.29\% and 3.26\% extraction efficiency. Then phytochemical analyses were performed on the extracts to investigate the presence of tannins, flavonoids, flavonones, flavonols, catechins and xanthones. The antioxidant activity of extracts was determined by in vitro photocolorimetric method based on the sequestration of free radicals, using DPPH (1,1-diphenyl-2-picryl-hydrazyl). Both extracts were able to sequestrate DPPH free radical. Croton blanchetianus extract activity showed to be more efficient than BHT (Butylated Hidroxytoluene) (positive control), with $\mathrm{EC}_{50}$ $=6.5 \pm 0.5 \mathrm{mg} \mathrm{mL}^{-1}$. Croton heliotropiifolius extracts showed lower antioxidant activity, with $\mathrm{EC}_{50}=50.15 \pm 0.21$ but still higher than that observed for BHT. However, the results observed for the ethanol extracts are possibly the result of the presence and concentration of phenolic compounds such as tannins and flavonoids, which were identified in this study.
\end{abstract}

Keywords: free radicals, phytochemistry, photocolorimetric.

\section{Introdução}

O organismo humano produz constantemente radicais livres por meio de suas atividades metabólicas. Apesar de ser um processo normal na vida dos organismos vivos, quando em excesso, podem gerar estresse oxidativo, levando a alterações 
teciduais responsáveis por diversas patologias, como artrites inflamatórias, úlceras e diabetes (El-Remessy et al., 2003), e processos degenerativos como doença de Alzheimer, doença de Parkinson, arteriosclerose e o envelhecimento precoce (Sikora et al., 2008) dentre outras. Para impedir os efeitos deletérios associados ao excesso dessas espécies reativas de oxigênio, o organismo apresenta defesas antioxidantes. No entanto, os antioxidantes são um grupo de substâncias que, quando presentes em concentrações ideais em relação aos substratos oxidáveis, reagem com os radicais livres impedindo ou diminuindo o estresse oxidativo (Nascimento e Rodrigues, 2007). Os antioxidantes presentes no organismo podem ser de origem endógena agindo enzimaticamente, como o superóxido dismutase (SOD), catalase (CAT) e glutationa peroxidase (GPx), ou não-enzimaticamente, como glutationa reduzida (GSH; Pimentel 2006).

Além destes antioxidantes produzidos no organismo, existem aqueles Dessa forma, o presente trabalho teve como objetivo sintéticos, substâncias utilizadas na indústria alimentícia, destacando-se o hidroxitolueno butilato (BHT), Butilhidroxianisol (BHA), propilgalato (GP), tercbutilhidroquinona (TBHQ) e os naturais tais como: $\alpha$-tocoferol (vitamina E), $\beta$-caroteno, ascorbato (vitamina C) e os compostos fenólicos (ácidos fenólicos e flavonóides; Sousa et al. 2007) os quais são os responsáveis pela remoção dessas espécies reativas.

O uso de antioxidantes sintéticos tem diminuído, devido a suspeita de atividades como promotores de carcinogênese, bem como devido à rejeição de aditivos sintéticos em alimentos (Bakkali et al., 2008). Nesse contexto, a procura por substâncias antioxidantes naturais tem aumentado nas últimas décadas, em especial os produtos naturais extraídos de plantas medicinais.

Os antioxidantes vegetais são de natureza muito variada, mas os compostos fenólicos têm sido apontados como responsáveis por maior capacidade antioxidante, sendo representados pelos flavonoides e isoflavonóides, taninos, lignanas, xantonas e outros. A ação antioxidante da maioria dessas substâncias naturais se deve a presença de hidroxilas fenólicas e as suas propriedades de oxirredução, pois eles agem como agentes redutores, doadores de hidrogênio. Além desses compostos, vários outros com atividade antioxidante tem sido isolado de diversas famílias de plantas (Fonseca et al., 2009, Rebelo et al., 2009).

De acordo com a literatura, estudos mostram que tanto óleos essenciais (Souza et al., 2007) como componentes não voláteis (extratos), extraídos de plantas (Balestrin et al., 2008, Nunes et al., 2008), têm sido estudados quanto à a avaliação do seu potencial antioxidante, demonstrando alta eficiência.

Dessa forma, o presente trabalho tem como objetivo identificar as principais classes de metabólitos secundários presentes nos estratos etanólicos das folhas de $C$. blanchetianus e $C$. heliotropiifolius bem como avaliar o seu potencial antioxidante pelo ensaio de captura de radicais DPPH.

\section{Material e Métodos}

As folhas de $C$. blanchetianus e $C$. heliotropiifolius foram coletadas nos meses de outubro e novembro de 2009 às 08:00 horas, no sítio São José do Bonfim - município de Patos, entre as coordenadas geográficas de latitude de $07^{\circ} 08^{\prime} 209^{\prime \prime}$ e longitude de $037^{\circ} 18^{\prime}$ 062". Em seguida uma amostra representativa de cada espécie foi identificada e depositada no Herbário CSTR da Universidade Federal de Campina Grande - UFCG, Patos-PB, sob o registro de número \#496 e \#497, respectivamente.

Os extratos etanólicos das folhas foram obtidos pelo método de extração exaustiva a frio (Matos, 1997). As folhas frescas de C. heliotropiifolius (300g) e C. blanchetianus $(280 \mathrm{~g})$ foram previamente trituradas e submetidas à extração com etanol durante 72 horas. Após o período de extração, o solvente foi destilado em evaporador rotatório a $80^{\circ} \mathrm{C}$ sob pressão reduzida. Os extratos etanólicos brutos obtidos foram pesados e armazenados a temperatura ambiente até a realização das análises fitoquímicas e ensaios antioxidantes. Foram realizados testes fitoquímicos para identificar as principais classes de metabolitos secundários nos extratos vegetais de acordo com a metodologia descrita por (Matos, 1997), que é baseado na mudança de cor ou formação de precipitados após a adição de reagentes específicos.

A atividade antioxidante dos extratos etanólicos das folhas foi determinada pelo método fotocolorimétrico in vitro realizada por meio do sequestro de radicais livres, usando o DPPH (Mensor et al., 2001). Essa análise é baseada na habilidade de compostos em doar um próton para o radical DPPH e formar estruturas de ressonância estáveis, estabilizando assim o radical livre.

As amostras para a realização do ensaio foram preparadas adicionando-se $1 \mathrm{~mL}$ da solução de DPPH $(60 \mu \mathrm{M})$ em $2,5 \mathrm{~mL}$ de soluções dos extratos que foram diluídas em etanol nas concentrações de $5,10,25,50,125 \mu \mathrm{g} \mathrm{mL}^{-1}$, em triplicata.

A solução de DPPH possui uma coloração roxa intensa e a atividade antioxidante de uma amostra pode ser visualizada pelo progressivo descoloramento da solução de DPPH, ao final do qual a mesma torna-se amarelada (Nunes et al., 2008). Após o tempo de reação de 30 minutos das amostras preparadas, as absorbâncias foram lidas com auxílio de Espectrotofotômetro de Ultravioleta UV-Vis com comprimento de onda ajustado para $520 \mathrm{~nm}$.

Um teste em branco foi realizado adicionando-se $1 \mathrm{~mL}$ de etanol a $2,5 \mathrm{~mL}$ das concentrações dos extratos. Como controle negativo foi usada a mistura de $1 \mathrm{~mL}$ da solução de DPPH com 2,5 mL de etanol e como controle positivo utilizou-se o 2,5 mL das concentrações de BHT (butilhidroxitolueno) e $1 \mathrm{~mL}$ da solução de DPPH.

Todas as leituras foram realizadas em triplicata e, com a média dos dados obtidos foi calculada a diferença de absorbância entre as amostras e o controle negativo, sendo as atividades antioxidantes (AA) percentuais obtidas por regressão linear, para cada fase, chegando-se assim à concentração das amostras que promovesse a diminuição para a metade da concentração inicial de DPPH (50\%), definida como Concentração Efetiva ( $\mathrm{CE}_{50}$; Mensor et al., 2001).

A atividade antioxidante (AA) das amostras por sequestro do DPPH foi expressa em percentagem, considerando as absorbâncias (Abs), segundo a equação: AA\% $=100-\{[$ (Abs extrato - Abs branco) x 100] / Abs controle\}.

As análises estatísticas das médias em triplicata $(n=3) \pm$ 
desvio padrão foram realizadas usando a Análise de Variância (ANOVA) seguida pelo teste de Student-Newman Keuls (SNK) para a múltipla comparação. Os resultados com p < 0,05 foram considerados significantes. Todas as análises foram realizadas usando o programa software GraphPad Prism 5.

\section{Resultados e Discussão}

As massas do material vegetal seco utilizado e dos extratos etanólicos obtidos da percolação, assim como os rendimentos em extrativos constam na Tabela $1 . \mathrm{Na}$ prospecção fitoquímica realizada com os extratos etanólicos das folhas de C. heliotropiifolius e C. blanchetianus foram identificados a presença de taninos condensados, flavonoides, flavononas, flavonóis, catequinas e xantonas (Tabela 2). A presença de catéquicos e flavonoides nas folhas de $C$. heliotropiifolius está de acordo com as descrições feitas por (Randau et al., 2004).

Tabela 1. Dados relacionados à obtenção dos extratos etanólicos das folhas $C$. heliotropiifolius e C. blanchetianus.

\begin{tabular}{lllc}
\hline \multicolumn{1}{c}{ Espécie } & $\begin{array}{l}\text { Massa das } \\
\text { folhas }(\mathrm{g})\end{array}$ & $\begin{array}{c}\text { Massa do } \\
\text { extrato }(\mathrm{g})\end{array}$ & $\%$ \\
\hline C. heliotropiifolius & 300 & 9,87 & 3,29 \\
C. blanchetianus & 280 & 9,15 & 3,26 \\
\hline
\end{tabular}

$\mathrm{Na}$ análise dos resultados da atividade antioxidante (AA\%) frente ao radical DPPH, considerou-se como valor de referência a $\mathrm{CE}_{50}$ de $35,50 \pm 0,50 \mu \mathrm{g} / \mathrm{mL}^{-1}$ do BHT (controle positivo) para comparar com a atividade antioxidante dos extratos etanólicos das folhas frescas de $C$. heliotropiifolius $e$ C. blanchetianus, pois o BHT é bastante utilizado como padrão para a atividade antioxidante (Mensor et al., 2001). No mesmo sentido (Melo et al., 2010), descreveram em seu estudo no Brasil, utilizando o método DPPH, 14 espécies de plantas com atividade antioxidante, reduzindo a concentração de radicais livres em $50 \%\left(\mathrm{CE}_{50}\right)$.

Tabela 2. Classe de metabólitos secundários identificados por prospecção nos extratos botânicos das folhas de $C$. blanchetianus e C. heliotropiifolius.

\begin{tabular}{|c|c|c|}
\hline Metabólito Secundário & C. blanchetianus & C. heliotripiipiifolius \\
\hline Taninos & $*$ & $*$ \\
\hline Fenóis & - & - \\
\hline Antocianinas e & & \\
\hline Antocianidinas & - & - \\
\hline $\begin{array}{l}\text { Flavonas, Flavonóis e } \\
\text { Xantonas }\end{array}$ & - & $* * *$ \\
\hline Chalconas e Auronas & - & - \\
\hline Flavanonóis & * & - \\
\hline Leucoantocianidinas & - & - \\
\hline $\begin{array}{l}\text { Catequinas (Taninos } \\
\text { catéquicos) }\end{array}$ & $*$ & $*$ \\
\hline Flavanonas & $*$ & * \\
\hline
\end{tabular}

(*) Presença de compostos; (**) Presença moderada, (***) Forte presença, (-) Ausência de compostos.

$\mathrm{Na}$ Tabela 3 estão representados os resultados quantitativos da atividade antioxidante dos extratos etanólicos das plantas.
Portanto, observa-se que os extratos apresentaram atividade antioxidante como sequestradores do radical livre DPPH, sendo que o extrato de C. blanchetianus demonstrou uma maior atividade (isto significa um menor valor de $\mathrm{CE}_{50}$ ) em comparação ao BHT, com $\mathrm{CE}_{50}$ de $6,5 \pm 0,5 \mu \mathrm{g} / \mathrm{mL}^{-1}$. O extrato das folhas $C$. heliotropiifolius mostrou menor atividade antioxidante, com o valor da $\mathrm{CE}_{50}$ de 50,15 \pm 0,21 superior ao observado para o BHT.

Tabela 3. Resultados da atividade antioxidante dos extratos etanólicos das folhas de $C$. heliotropiifolius e $C$. blanchetianus utilizando o radical DPPH.

\begin{tabular}{cccc}
\hline Concentração & EFCb & EFCh & BHT \\
\hline 5 & $34,42 \pm 2,38$ & $9,35 \pm 3,78$ & $3,30 \pm 0,06$ \\
10 & $73,81 \pm 2,57$ & $13,56 \pm 1,85$ & $10,52 \pm 0,11$ \\
25 & $88,65 \pm 1,35$ & $28,72 \pm 1,6$ & $31,82 \pm 0,22$ \\
50 & $98,12 \pm 1,85$ & $48,43 \pm 8,15$ & $60,12 \pm 0,11$ \\
125 & $93,29 \pm 0,96$ & $92,06 \pm 0,5$ & $88,03 \pm 0,49$ \\
CE $50 \%$ & $6,5 \pm 0,5$ & $50,15 \pm, 0,21$ & $35,50 \pm 0,50$ \\
\hline * EFCb:
\end{tabular}

* EFCb: Extrato das folhas de C. blanchetianus; EFCh: Extrato das folhas de C. heliotropiifolius. BHT: Butil hidroxitolueno.

A diferença na atividade antioxidante observada para os extratos etanólicos das espécies em estudo, possivelmente seja atribuída à presença e a concentração de compostos fenólicos pertencentes a classes dos taninos e flavonóides verificados nos extratos, cuja ação antioxidante é conhecida na literatura. No entanto, a atividade antioxidante desses compostos fenólicos tem sido atribuída às suas propriedades de óxidoredução, que desempenham importante papel na absorção ou neutralização de radicais livres (Basile et al., 2005).

Esses compostos derivados de plantas medicinais com atividades antioxidantes tem sido isolado das mais diversas famílias de plantas (Boudet, 2007, Razavi et al., 2008) e os flavonóides são, por certo, as substâncias representativas desta atividade (Van Den Berg et al., 2000), uma vez que possuem esqueleto carbônico propício para a estabilização de radicais livres. Contudo, a intensidade da ação antioxidante exibida por estes fitoquímicos é diferenciada, principalmente devido ao número e posição de hidroxilas presentes nas moléculas (Melo et al., 2008).

A atividade antioxidante tem sido descrita em algumas espécies de Croton, como C. celtidifolius (Nardi et al., 2003), C. nepetaefolius (Morais et al., 2006) e o C. argyrophylloides (Catunda Jr. e Morais, 2002). No entanto, ainda não há relatos de estudos do potencial antioxidante com extratos das espécies de $C$. blanchetianus e $C$. heliotropiifolius, somente com óleos essenciais.

Contudo, esses estudos são preliminares e servem como ponto de partida para estudos posteriores como o isolamento, a purificação e a elucidação estrutural das substâncias que funcionam como antioxidantes, além de ajudar a inferir sobre um possível mecanismo de ação dessas substâncias, finalizando por testar a viabilidade terapêutica em antioxidantes para seu uso futuro.

\section{Conclusões}

Os estudos com os extratos botânicos e $C$. heliotropiifolius e $C$. blanchetianus resultaram na comprovação de atividade 
antioxidante; muito provavelmente essa atividade é atribuída à presença de compostos fenólicos identificados na análise fitoquímica dos extratos etanólicos de ambas as plantas, principalmente taninos e flavonoides.

\section{Agradecimentos}

Agradecemos ao Conselho Nacional Científico e Tecnológico $(\mathrm{CNPq})$ pela bolsa concedida e o auxílio para execução do projeto.

\section{Referências}

Venancio, A.M. 2006. Toxicidade aguda e atividade antinociceptiva do óleo essencial do Ocimum basilicum L. (manjericão), em Mus músculos (camundongos). Dissertação de Mestrado, Universidade Federal de Sergipe, Aracaju. $110 \mathrm{f}$

Bakkali, F.; Averbeck, S.; Averbeck, D.; Idaomar, M. 2008. Biological effects of essentials oils: A review. Food and Chemical Toxicology. 46: 446475.

Basile, A.; Ferrara, L.; Del Pozzo, M., Mele, G.; Sorbo, S.; Bassi, P.; Montesano, D. 2007. Antibacterial and antioxidant activities of ethanol extract from Paullinia cupana Mart. Journal of Ethnopharmacology. 102: 32-36.

Boudet, A.M. 2007. Evolution and current status of research in phenolic compounds. Phytochemistry. 68: 22-24.

Balestrin, L.; Dias, J.F.G.; Miguel, O.G.; Dall'Stella, D.S.G.; Miguel, M.D. 2008. Contribuição ao estudo fitoquímico de Dorstenia multiformis Miquel (Moraceae) com abordagem em atividade antioxidante. Revista Brasileira de Farmacognosia. 18: 230-235.

Catunda-Junior, F.E.A.; Luciano, J.H.S.; Morais, S.M. 2002. Atividade antioxidante de óleos essenciais de plantas do Nordeste do Brasil. Revista de Ciência \& Tecnologia, 4:. 23-29.

EL-Remessy, A.B.; Behzadian, M.A.; Abou-Mohamed, G.; Franklin, T.; Caldwell, R.W.; Caldwell, R.B. 2003. Experimental diabetes causes breakdown of the blood-retina barrier mechanism involving tyrosine nitration and increases in expression of vascular endothelial growth factor and urokinase plasminogen activator receptor. American Journal of Pathology, v.162, p. 1995-2004.

Fonseca, A.M.; Bizerra, A.M.C.; Souza, J.S.N.; Monte, F.J.Q.; Oliveira, M.C.F.; Mattos, M.C.; Cordel, G.A.; Braz-Filho, R.; Lemos, T.L.G. 2009. Constituents and antioxidant activity of two varieties of coconut water (Cocos nucifera L.). Revista Brasileira de Farmacognosia, 19:193198.

Matos, F.J.A. 1997. Introdução à fitoquímica experimental, 1 ed. UFC,. 141p.

Mensor, L.L.; Menezes, F.S.; Leitão, G.G.; Reis, A.S.; Dos Santos, T.C.; Coube, C.S.; Leitão, S.G. 2001. Screnning of Brasilian plant extracts for antioxidant activity by the use of DPPH free radical method. Phytother Research, 15: 127-130.

Morais, S.M.; Catunda-Junior, F.E.A.; Silva, A.R.A.; Stone, J.; Neto, M. 2006. Atividade antioxidante de óleos essenciais de espécies de Croton do nordeste do Brasil. Química Nova, 29: 907-910.

Melo, E.A.; Maciel, M.I.S.; Lima, V.L.; Nascimento, R.J. 2008. Capacidade antioxidante de frutas. Revista Brasileira de Ciências Farmacêuticas. 44: 193-201.

Melo, J.B.; Araújo, T.A.S.; Castro. V.T.N.A.; Cabral, D.L.V.; Rodrigues, M.D.; Nascimento, S.C.; Amorin, E.L.C.; Alburquerque, U.L. 2010. Antiproliferative Activity, Antioxidant Capacity and Tannin Content in Plants of Semi-Arid Northeastern Brazil. Molecules. 15, 8534-8542.

Nascimento, P. F.; Rodrigues, C. S. 2007. Atividade antimicrobiana dos óleos essenciais: uma abordagem multifatorial dos métodos. Brazilian Journal of Pharmacognosy. 17: 305-311.

Nardi, G.M.; Felippi, R.; Dalbos, S.; Siqueira-Junior, J.M.; Arruda, D.C.; Delle-Monache, F. 2003. Anti-inflammatory and antioxidant effects of Croton celtidifolius bark. Phytomedicine. 10: 176-184.

Nunes, X.P.; Mesquita, R.F.; Silva, D.A.; Lira, D.P.; Costa, V.C.O.; Silva, M.V.B.; Xavier, A.L., Diniz, M.F.F.M.; Agra, M.F. 2008. Constituintes químicos, avaliação das atividades citotóxica e antioxidante de Mimosa paraibana Barneby (Mimosaceae). Revista Brasileira de Farmacognosia. 18: $718-723$.
Pimentel, F.O. 2006. Atividade antioxidante de Byrsonima crassa Nied. e Byrsonima fagifolia Nied. em modelos de indução de úlcera gástrica.. Dissertação de Mestrado em Farmacologia. Universidade Estadual de Campinas, SP. 111p.

Randau, K.P. 2004. Estudo Farmacognóstico de Croton rhamnifolius H.B.K. e Croton rhamnifolioides pax \& hoffm. (Euphorbiaceae). Revista Brasileira de Farmacognosia. 14: 89-96.

Razavi, S.M.; Nazemiyeh, H.; Hajiboland, R.; Kumarasamy, Y.; Delazar, A.; Nahar, L.; Saker, S.D. 2008. Coumarins from the aerial parts of Prangos uloptera (Apiaceae). Revista Brasileira de Farmacognosia. 18: 1-5.

Rebelo, M.M.; Silva, J.K.R.; Aandrade, E.H.A.; Maia, J.G.S. 2009. Antioxidant capacity and biological activity of essential oil and methanol extract of Hyptis crenata (Pohl) ex Benth. Revista Brasileira de Farmacognosia. 19: 230-235.

Sousa, C.M.; Silva, H.R.E.; Vieira-Junior, G.M.; Ayres, M.C.C.; Costa, C.L.S., Araujo, D.S.; Cavalcante, L.C.D.; Barros, E.D.S.; Araújo, P.B.M.; Brandão, M.S.; Chaves, M.H. 2007. Fenóis totais e atividade antioxidante de cinco plantas medicinais. Química Nova. 30: 351-355.

Souza, T.J.T.; Apel, M.A.; Bordignon, S.; Matzenbacher, N.I.; Zuanazzi, J.A.S.; Henriques, A.T. 2007. Composição química e atividade antioxidante do óleo volátil de Eupatorium polystachyum DC. Revista Brasileira de Farmacognosia. 17: 368-372.

Sikora, E.; Cieslik, E.; Leszczynska, T.; Filipiak-Florkiwuacz, A.; Pilsulewski, P.M. The antioxidant activity of selected cruciferous vegetables subjected to aquathermal processing. Food Chemistry. 107: 50-55, 2008.

Van Den Berg, R.; Haenen, G.; Van Den Berg, H.; Vander, V.W.; Bast, A. 2000. The predictive value of the antioxidant capacity of structurally related flavonoids using the trolox equivalent antioxidant capacity (TEAC) assay. Food Chemistry. 703: 391-395. 